\title{
Discourse Analysis of Blizzard Entertainment's Marketing Communications
}

\author{
Catherine Tabequero
}

Faculty of Letters and Arts, Lucian Blaga University of Sibiu, Romania

Received August 19, 2019; Revised September 23, 2019; Accepted May 13, 2020

\section{Cite This Paper in the following Citation Styles}

(a): [1] Catherine Tabequero , "Discourse Analysis of Blizzard Entertainment's Marketing Communications," Advances in Economics and Business, Vol. 8, No. 6, pp. 333 - 345, 2020. DOI: 10.13189/aeb.2020.080603.

(b): Catherine Tabequero (2020). Discourse Analysis of Blizzard Entertainment's Marketing Communications. Advances in Economics and Business, 8(6), 333 - 345. DOI: 10.13189/aeb.2020.080603.

Copyright $\mathrm{C} 2020$ by authors, all rights reserved. Authors agree that this article remains permanently open access under the terms of the Creative Commons Attribution License 4.0 International License

\begin{abstract}
This study examines the nature of the discourse in advertising. The focus is on Blizzard Entertainment's Marketing Communications, which is directed towards the promotion of their products and service to the general public. The study aims at uncovering the basic elements of the most pervasive, influential and inescapable discourse of Blizzard Entertainment advertisements. Norman Fairclough's 3-D model of visual design was used to analyze the data. It focuses on the interaction of language, image and format and analyzes advertising persuasive strategies. Also, this study gives analyses of certain ads, utilizing various methods of interpretations and ends with a discussion on advertising discourse. In this connection, the analyses are provided with a perspective on demonstrating the advertisements develop importance through collaboration with different kinds of discourse, and how it is indistinguishable from social and cultural discourse. Thus, it discusses Blizzard Entertainment's advertisements influence and on how these advertisements have an impact on our society and cultural values. The results of this descriptive study serve as a support and of great help to the future researches related to social media and advertising.
\end{abstract}

Keywords Advertising, Discourse Analysis, Marketing

\section{Introduction}

Advertising is mass media content intended to persuade audiences, readers, viewers or listeners to take an action on products, services and ideas (Arroyo, 2014). The idea is to drive consumer behavior with a certain goal in mind as to a product, service or concept. However, some different researchers disagree, Cook (1992) states that advertisements can amuse, inform, misinform, stress or warn. Advertising is so familiar to modern readers that it may seem odd to ask what an advertisement is. Although advertising is all around us - perhaps because it is all around us - we don't often pause to think about its nature as a form of discourse, as a system of language use whereby, on a daily basis, huge numbers of readers have fleeting 'conversations' with the writers of countless texts (Goddard, 2001).

Advertisements can be utilized to refer to text, audio, video, photography and graphic designs. Advertising can be channeled through numerous tubes like newspapers, magazines, radio and television broadcasts, stage shows, websites, bulletins, posters, wall paintings, vehicles, and so forth. Actually, advertisers more often than not have full control of the message all the way to the audience (Arroyo, 2014). However, for an advertisement to be effective, it ought to have smart placement where it will be seen by the target interest group; an enticing feature, which is the most significant specialized part of the advertisement; a persuasive headline which suggests benefits or news.

Readers scan headlines, so if yours does not catch an eye, it won't be read. Eye-getting graphics are also significant means of getting your audience to read the advertisement. An appealing graphic and a solid headline will pull a reader or viewer into the advertisement. Concentrating on the goal is required to persuade customers to satisfy your one main objective. Having various objectives will confuse people and after that they will not get the message. Tempting offer 
including valuable bonuses and risk free, easy pay terms, and low cost will help in promoting the product; it will support purchasers reconcile the offer in their minds, so it should make sense and be authentic.

The focus of analysis in this study is the Blizzard Entertainment advertisement in blogs, and social media. We discovered it as a striking product of advertising to exemplify how Blizzard Entertainment discover approaches to inspire and persuade individuals to buy their products. To do so, they use language wisely in light of the fact that as Foucault (1975) proposed, extraordinary guarantees are best showed with language. Indeed, a standout amongst the most efficient media tools is TV advertisements because almost each individual reads blogs and uses social media and is exposed to various advertisements (Okan, 2017). They are perhaps awed by the sounds, images, basic conversations, mottos, excellent colors and, obviously, by the discursive power of language utilized in advertisements.

Thus, to analyze the advertisements fundamentally is a necessity to uncover inclinations, hidden messages or manifestations which are included within entrancing words. The researcher worked with English language advertisements posted in blogs and social media because advertisement is based on Blizzard Entertainment image and there is a need to uncover, with their points of view, what kind of messages the advertiser attempts to convey on to the viewers with this image created. As such, the aim is to see how they respond to ideologies and manipulations imposed in the advertisement. Needless to say, those ideologies and manipulations are unavoidably under the influence of the linguistic preferences made by the advertiser.

Therefore, the intent is to highlight how Blizzard Entertainment interpret those linguistic preferences and how they construct their very own understanding by basically analyze the language of the advertisement. That is, the researcher hopes this will help Blizzard Entertainment raise their awareness of the power of language in social practices and pass on this awareness to consumers and how their advertisements affect the social and cultural values of every individual.

\section{Materials and Methods}

This study will address the following research questions:

\subsection{Research Questions}

1. What are the linguistics devices used in Blizzard Entertainment's Marketing Communications?

2. How does the language of advertisements influence people?

3. Do these advertisements have an impact on our society and cultural values?

\subsection{Theoretical Framework}

The theoretical framework employed in this study is the Three-dimensional Model approached by Fairclough (1995). Advertisements are seen as media discourse as they involve language and social processes. Hence, this model is used to show the link between the nature of social practice and the properties of language "texts".

\subsection{The Three-dimensional Model}

The Three-dimensional model, approached by Fairclough (1995), is applied as theoretical framework for the research. According to Fairclough (1975), discourse that happens in any context ought to be viewed as a language text which can be either spoken or written; discourse practice which is related to production and interpretation of text and sociocultural practice. The discourse is profoundly associated with different levels socio cultural practice. From the individual situation the relation to the more extensive level can be denoted.

\subsection{Text Analysis}

Text analysis is, obviously, a simplistic definition, and one which will be intensely refined through the course of the year. If we want to comprehend the role that the media play in our lives and accurately how its messages participate in the cultural construction of our perspective on the world, then we need to understand what meanings audiences are making of TV programs, of films, of newspapers, magazines, and radio programs - to put it shortly, of 'texts' (Mckee, 2003).

Text analysis is comprised with both the form of the text and the meaning. Flairclough (1992) proclaimed that it is a perplexing process to analyze a text in the basis of meaning as texts are ambivalent in character and can be interpreted in different ways. Moreover, both the potential meaning of form and interpretation are required in text analysis. He added that text analysis has four main features, 'vocabulary', grammar, cohesion, and text structure'. Here, images are also included in the textual analysis.

\subsection{Discursive Practice}

Production, distribution and consumption of text are collectively known as discursive practice. The relation between the text producers with the context of the production is also focused in discursive practice. Discursive practice additionally focuses around the consumption of the text. It implies how the text is considered by the receiver. It can also detect the social identities of individual or of groups. The context of the discourse controls over the text interpretation.

In simpler terms, according to Gee (1999), by a "practice" or a socially perceived and institutionally or culturally upheld endeavor that typically includes 
sequencing or consolidating activities in certain predetermined ways. Empowering a student is an action, mentoring the student as his or her advisor in a graduate program is a training. Enlightening somebody something regarding linguistics is an activity (educating), addressing on linguistics in a course is a practice. Sometimes the term "activity" is utilized for what I am known as a practice.

\subsection{Discourse as Social Practice}

Discourses are forms of social practice. They are also obviously texts (in the wider sense of the word). But Fairclough's framework adds a "mediating" third dimension "which focuses on discourse as a specifically discursive practice" (Fairclough, 1992).

Discursive practice is itself a form of social practice, and focuses on the process of text production, distribution and consumption (Lawson, 2008). Fairclough portrays this framework as "an attempt to unite three analytical traditions, each of which is crucial for discourse analysis". These analytical traditions are:

- The tradition of close textual and linguistic analysis within linguistics.

- The macro sociological tradition of analyzing social practice in connection to social

- $\quad$ structures.

- The interpretivist or micro sociological tradition of seeing social practice as something which people actively produce and make sense based on shared commonsense procedure.

Discourse in social practice demonstrates the connection of discourse with ideology and power. Ideologies are indications of the real world. The realities connected with the physical world, social relations and social identities. These realities are based upon various levels of forms or meanings of discursive practices. These impact production, reproduction and transformation of relation. Discursive practice with regards to various context transforms into ideologies and after that making the domination gets the power. There is dependably a battle between ideologies which use discourse in various levels of power exercising.

\section{Results and Discussion}

The analysis is presented at the textual and social practice level based on Flairclough's Model.

\subsection{What are the Linguistics Devices Used in Blizzard Entertainment's Marketing Communications?}

A. Analysis of Printed Ads:

1. Use of Fonts

Use of various fonts is firmly perceptible in the print advertisements. Unique information of the service and the packages are written in various fonts. Some information is written in so small fonts that they are not really readable. According to Goddard (2001), utilizing different fonts and typographies are attention seeking devices and use of various fonts makes a special effect.

In printing the management, with the advancement of computer Technologies, just about an infinite number of fonts were created. Every font has its very own language. Some fonts are generally reasonable with a wide range of writing and telling, some are just eligible to give certain messages. Still, some fonts are intended for writing though some are just of a decorative nature. (Heller and Ilic, 2012:62).

When the wrong font is used, it is probably going to cause misunderstandings and wrong messages being sent. Unconsciously and insensibly chosen fonts both make the writing harder to read and may give the wrong message. A font chosen only for the beauty of its aesthetic sight might be a deterrent as it is hard to read (Nakilciuglu, 2013).

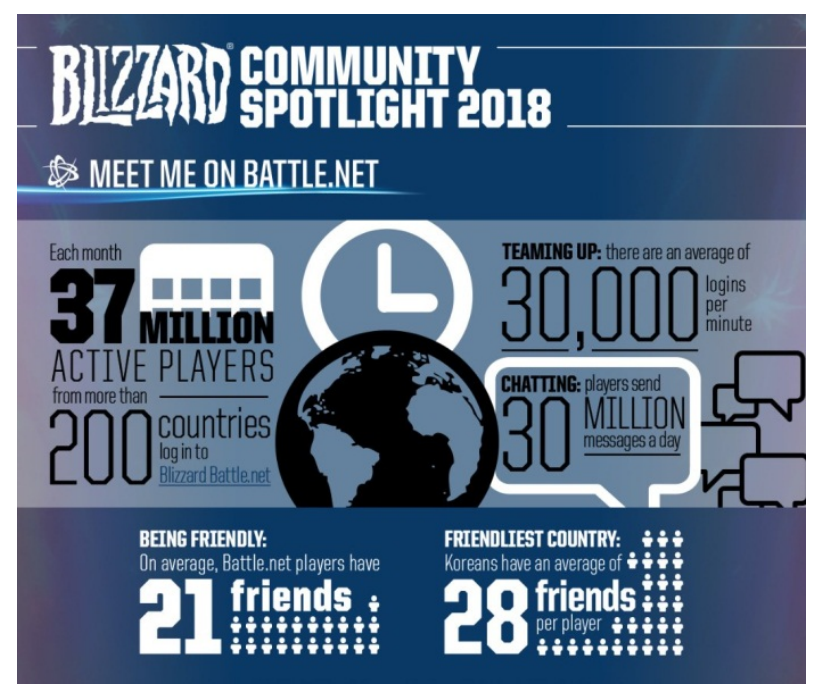

Figure 1. Use of different 'Font' as attention seeking device

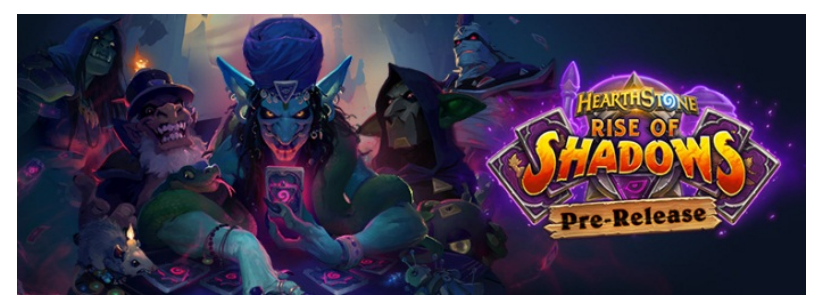

Figure 2. Use of different 'Font' as attention seeking device

It tends to be seen that the advertisement has utilized different font sizes and colors to catch the attention of the of people. It is also seen that it is very creative and pleasing to the eyes. Thus, fonts are playing a part in the promotional advertisements for marketing. The usage of fonts is like an art of arranging type to make written language legible, readable and appealing when displayed.

In the first figure, there are small fonts used. People 
hardly read that part since it is written in small little fonts. People tend to ignore the information not deliberately but rather unconsciously, which are written in small font as it doesn't attract people as much as much as the big colored fonts. It is strategies followed by the promotion creators to make the audience not to see all the written information (Dasgupta, 2015).

\section{Word Play}

Playing with words is remarkable in the advertisements. The creative writers try to input some new things to get the attention of the audience. As advertisement is also entertaining, so the words with various formation entertain the audience and also effectively achieve the attention of the customer.

"Wordplay (or pun) is a rhetorical device that frequently depends on the distinctive implications of a polysemic word, the literal and non-literal meaning of an idiom or on bringing two homonyms together in the same utterance to produce a witticism.

Punning is often utilized in commercial advertising to pull in the reader's attention and looking after her/his interest for keeping with the AIDA rule" (Lund 1947, 83). The AIDA principle is basic for the entire field of advertisement and in this work it has been already mentioned regarding idioms. The words hidden behind the abbreviation are Attention, Interest, Desire and Action. All the figures of speech mentioned before are in fact more than able to successfully contribute to coming to AIDA principle (Korcak, 2012). Furthermore, puns are even reasonable for promotion since they are hilarious, clever and also noteworthy. These features of puns on words are viewed as the absolute most valuable ones in the field of advertising (Laviosa 2005).

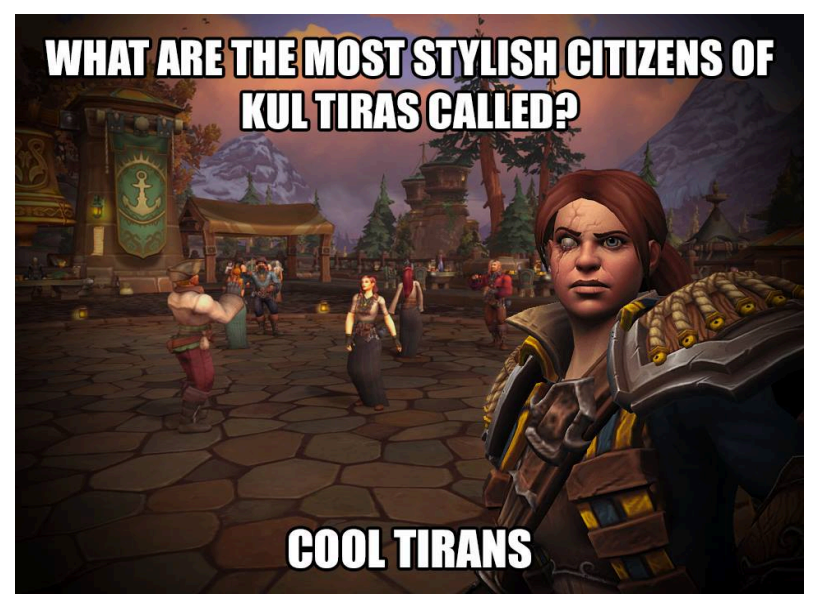

Figure 3. Word Play

To make the language interesting the spelling of the word "Kul Tiras" is written or changed in a unique way. This word play is clever, tight, and stylized in a way that they can attract the idea.

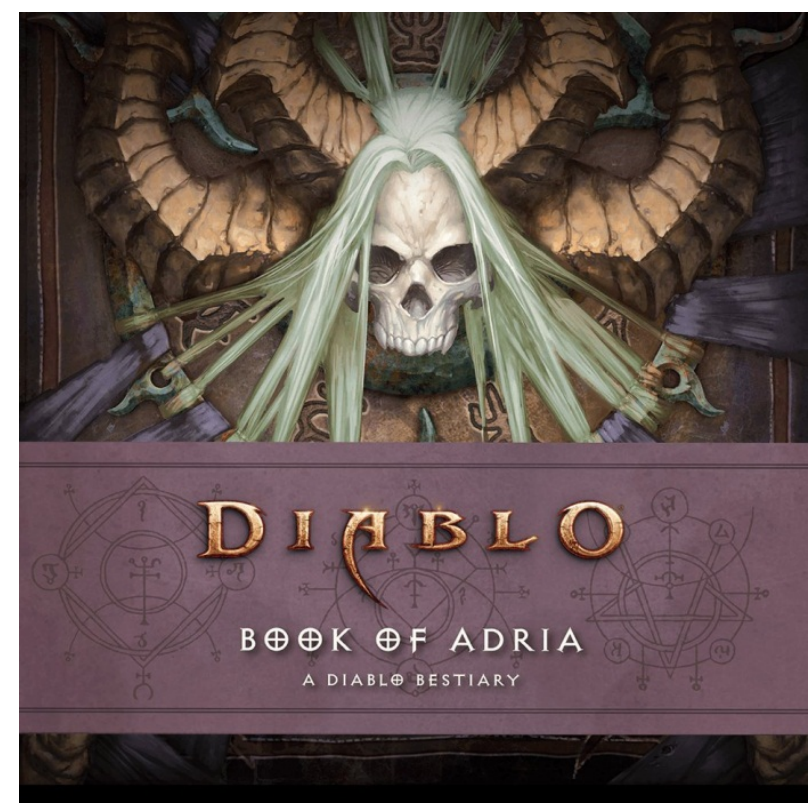

Figure 4. Word Play

Another remarkable word play that can be seen above is the "A Diablo Bestiary". Instead of using "Diary", they used "Bestiary". Thus, it infers with the descriptive and anecdotal treatise on various kinds of animals especially a medieval work with a moralizing tone.

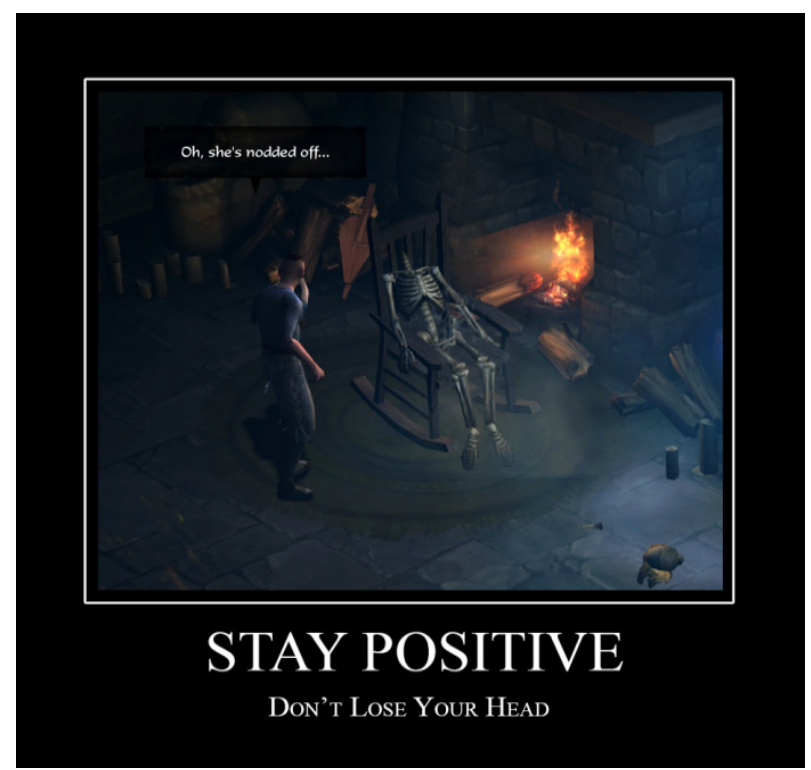

Figure 5. Use of Pun

The lines shown above "Oh, she's nodded off..." is dry humor but it attracts the attention of the customer. The "STAY POSITIVE" also gives a good impact to the customer as it is a good advice. However, the "Don't Lose Your Head" implies the positivity. It conveys a message to the people not to lose hope.

3. Ellipsis

Full structured sentences are not used in the 
advertisements most of the time. Some words are omitted keeping the words from which the message can be assumed.

Ellipsis or elliptical construction alludes to the omission from a clause of at least one word that would otherwise be required by the rest of the elements. Ellipsis is an implicit reference to some material, either previously mentioned, or some way or another inferable. Henceforth, ellipsis, being the outcome of the conventional aspect of the sentence, offers approach to semantic presupposition.

The term ellipsis has been connected to a wide scope of phenomena over the hundreds of years, from any circumstance in which words seem, by all accounts, to be missing, to a much smaller scope of specific constructions (Jawad, 2016). Ellipsis keeps on being of central interest to theorists of language precisely on the grounds that it speaks to a circumstance where the typical structure/which means mappings, the algorithms, structure, rules, and constraints that in non-elliptical sentences enables us to outline and gestures onto their corresponding meanings, break down. In fact, in ellipsis, the standard mapping appears to be completely missing. In ellipsis, there is a meaning without form (McCarthy, 1991:152).

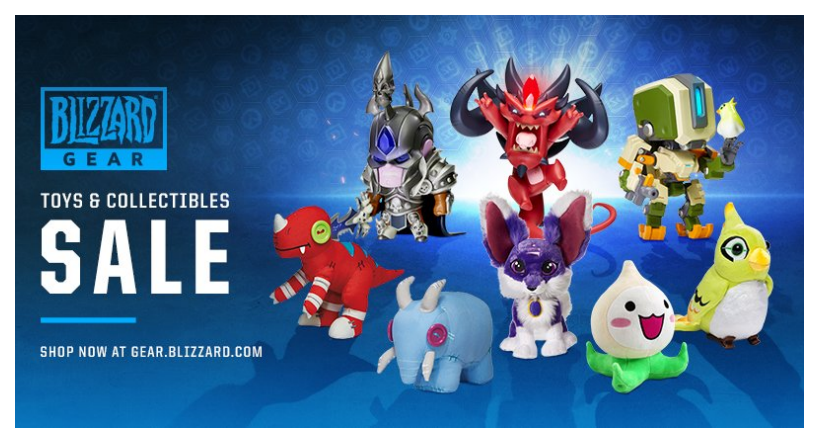

Figure 6. Ellipsis

As noticed, instead of using, "Toys and Collectibles for Sale" only "Toys and Collectibles - SALE" is used. This is to attract directly the attention of the customers about the sale. However, this doesn't break the proper grammar of English. It's just that there is a difference when it comes to the proper phrasing of it.

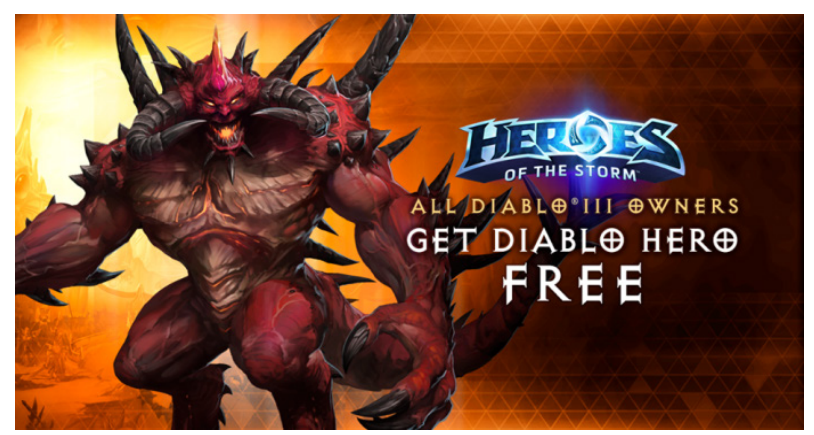

This is another example of ellipsis (the same as above). Instead of using "Get Diablo Hero for Free", they just used "Get Diablo Hero FREE". This is to attract customers directly to the word "FREE". Most of the time, when it comes to the advertising, advertisement creators omit some words and focus on the usage of fonts in order for the advertisement to be effective to the customer.

\section{Rhyme and Rhythm}

Rhyme is broadly perceived as being associated with poems. Rhyming is common in poetry and it essentially implies sounds agree with, and all the more frequently alludes to, end rhymes. The difficulty in leveraging rhyme as a feature is to determine the degree to which two words rhyme. The Moby pronunciation dictionary, from Carnegie Mellon University, gives a guide from words to phonemes, however this does not entirely solve the problem as robustly identifying rhyme requires operating at the syllable level rather than to at the phoneme level (Dara, et al., 2008).

Rhythm is a fundamental part of life. In humans, our ability to create and perceive rhythmic behavior, for example in music and language, may have developed from primates (Fitch et al. 2005), or may be interestingly human (Pinker and Jackendoff 2005). Non-experts would most likely perceive the term rhythm, yet scholarly writing on the phenomenon since ancient times has not given one simple definition, for two fundamental reasons (Cumming, n.d.). To begin with, rhythm shows itself in different forms (Adams 1979). Second, the idea of rhythm to which researchers in each field refer is perplexing with a few variables (Fraisse, 1982).

Rhyme and rhythm are also used in the advertisements to give a lyrical effect rhyme and rhythm are applied as linguistic device in the advertisements. Rhyme refers to sounds, not spelling. It is regularly found in jingles, slogans and headlines (Vasiloaia, n.d.)

The point of advertising is to be catchy and simple to recall. One of the devices how marketing specialists can achieve it is to utilize prosodic features - intonation, rhythm and lexical stress - on the grounds that they have an emotional and mnemonic impact. Copywriters frequently use language with rhythmical arrangement. The audience or the reader need not see it and he sees it just intuitively. The outcome is that the text is memorable and linguistically perfect. If the rhythm has some regularity, it is called meter. Meter is an example made out of rhythm groups (feet) comprising of similar or indistinguishable patterns of stressed and unstressed syllables. (...) Metrical scheme may effectively pass unnoticed.)

Figure 7. Ellipsis 


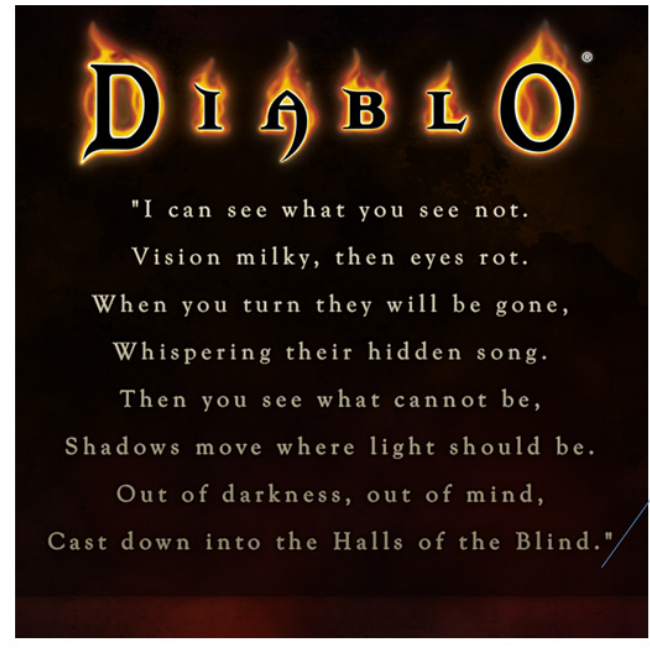

Figure 8. Rhyme and Rhythm

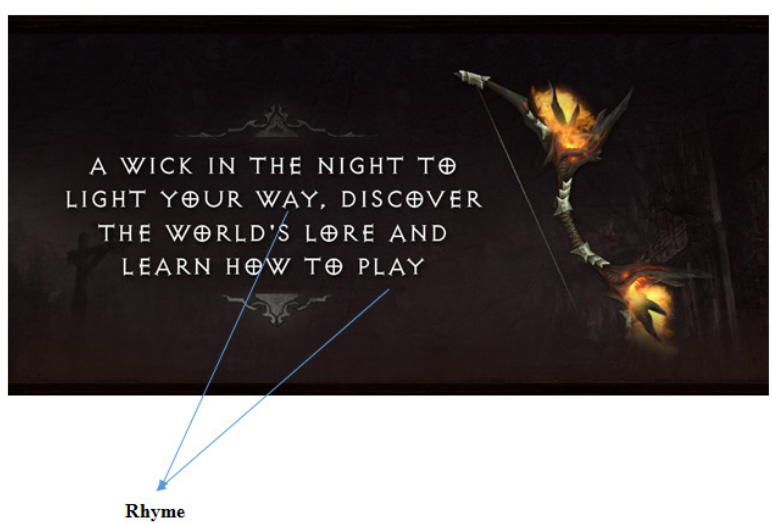

Figure 9. Rhyme and Rhythm

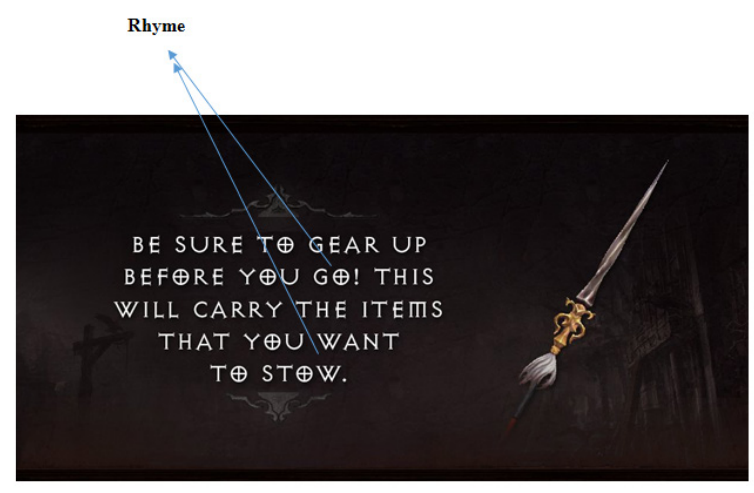

Figure 10. Rhyme and Rhythm

Most of the lines used give a lyrical effect to the advertisement. The attractive rhythmic words infer messages very quickly as it can have the attention of the consumer without difficulty. This is also very creative as they really create a very creative poem based on 'Diablo'. "Rot" and "not", "mind" and "blind" and even the word "be" create a very good rhyme that gives a lyrical sound in the poem. Even to the words, "way", "play", and "go", "stow".
People should use rhyme in business or personal persuasion. To start with, similar to any verbal tool, a little goes far. One subtle rhyme in a slogan or a catchphrase will make that phrase more believable just like what the figures 9 and 10. A rhyme could emphasize a product benefit or answer a typical complaint. You don't want your entire pitch to be in verse - that will make the audience to focus more on the mechanics of the delivery and wording instead of the message itself.

\section{Riddle Discourse}

Riddling is an exchange of words in which individuals are deliberately misdirected in light of the fact that the "right" answer is sometimes completely unexpected (Bregenhoj, 2018). Riddling is not, however, a general knowledge quiz. Apparently satisfying the criteria in the riddle question does not necessarily yield the guesser the pleasure of a right answer. In the riddling game, with rules from random analogy, arriving at the right answer is most often arbitrary. Riddles have been described from numerous points of view, and their definition relies upon their components, sometimes called "parts". A riddle is a conventional, fix-phrased verbal expression comprising of two parts, an image and an answer, and a seeming contradiction. For instance, "A house full, a yard full, couldn't get a bowl full. — Smoke". "Fix-phrased" ought to be understood in relation to "free phrased" genres of folklore, for example, folktales and, legends in which the content is more fixed than the real expression.

Consumer's involvement with advertisements diminishes as a result of the tremendous information-overload. Advertisers are, accordingly, challenged to devise new strategies to reach consumers. The strategy of intertextuality is frequently essential to the promotional success of a commercial or advertisement. The facts demonstrate that the message of an advertisement is bound to establish a connection on a customer if it contains more "attention value". As we have seen, there are various techniques accessible to the copywriter to accomplish this (Gully, 1996).

Riddles are a voyage into the unknown (Bregenhoj, 2001). They are an invitation to set out on an adventure that either brings enjoyment, entertainment and delight at finding the correct answer, or humiliation and vexation at being driven adrift. This is the reason why copywriters are using riddles in order to catch the attention of the consumer.

The copywriters are really creative in their marketing strategy. This riddle "A demon hides in the cloud, yet you can trap and take him in your pocket" is more of a clue that makes the audience dig and dig more for the answer. And this strategy makes the audience more interested into the game. Aside from the puns in the advertisements or posts, riddles, clues and some other mind blowing marketing 
strategies are gaining more attention to the audiences.

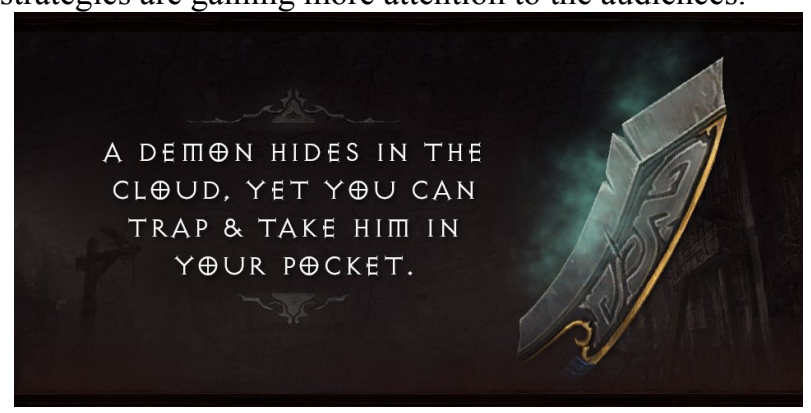

Figure 11. Riddle Discourse

\section{Time-Bound Greetings}

Despite the attention greetings have received in the social sciences, today there is no generalizable meaning of them and, in this way, no systematic way for deciding what qualifies as 'greetings' in a specific speech community (Shabeeb \& Jibreen, 2010). In any case, linguists have felt at ease recognizing greetings in various languages and giving hypotheses and definitions about what greetings accomplish for or to individuals.

Malinowski (1923) as cited in Nodoushan (2006) characterizes an expression of greeting, for example, 'How do you do' as an uncommon sort of speech that is called 'phatic communication' like a type of speech in which ties of union are created by a mere exchange of words". These words don't exchange meanings, rather, they satisfy a social function (Shabeeb \& Jibreen, 2010). Similar to such expressions are 'Nice day' and 'You are looking smart today'.

Time-bound greetings are those that are performed with specific reference to the temporal context amid which a conversation happens (Hakim et al., 2018). They are portrayed by the use of daily and seasonal greeting. They are encapsulated in such expressions as 'good morning', 'good afternoon', 'good evening', etc.

One convincing remark about time-bound greeting is that greeting expression itself determines the context of the utterance (Hakim et al., 2018). It is on the grounds that time-bound greeting will in general be performed with specific reference to the temporal context amid which a conversation happens. In this exchange, greeting utterance communicated by Replika alludes to a period of a day, at which the dialogue is going to start. Hence, time-bound greeting can't be performed purposely, suggesting that this particular type of greeting is intensely regulated by social routines.

The holiday season is a time of celebration. Celebration occurs when people do enjoyable or special things in honor of the holidays. Figure 12 is a time-bound greeting of Blizzard in honor of Valentine's Day. They posted it in their website with a caption "Happy Valentine's Day". It is a creative way of sending holiday greetings to the audience. Aside from its sweet message, it will also attract audience to what they are marketing as they put a background picture related to their games.

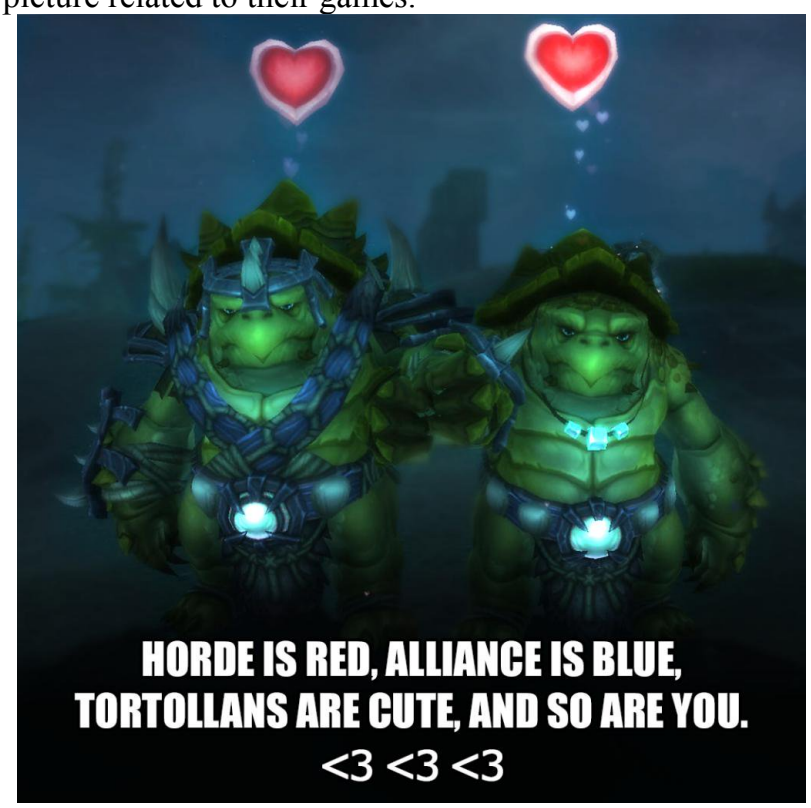

Figure 12. Time-Bound Greetings

Many companies are sending greeting cards as a marketing tool as they consider that this little gesture can say a lot about the way they do business and interact with their customers. Email and social media also are a nice way to send some holiday thoughts as it can have an impact in putting the business in the recipient's mind. Moreover, if they want to be remembered by the customers in a cheerful way, the message should be as important as a visual matter. In this case, figure 12 believes to capture the minds of the audience.

\section{Appreciation and Expression of Sympathy Post}

In the today's job market, the demand for top talent seems to be at an all-time high. Because of these, companies may feel tempted to put the greater part of their focus on recruiting specific candidates - or, to help maintenance, or rewarding top performers within the organization (Bull, 2019). However, companies shouldn't disregard the workers who probably won't be considered 'stars'.

While having standout workers can be a noteworthy differentiator in helping your organization accomplish its objectives, a company is, ultimately, just ever as solid as its group. That is the reason organizations that don't demonstrate the love to other workers might pass up a major opportunity.

On a general level, most employees need to feel the fulfillment of being recognized for the work they put into their job. The individuals who put their nose to the grindstone without acknowledgment from a supervisor or official may feel spurred to search for the door considerably more quickly than those whose efforts are called out regularly.

Employee recognition can come in numerous forms - 
and only one out of every odd employee needs to be recognized similarly, however don't belittle the positive attitude that a simple "thank you" can bring. Those two words, regardless of whether conveyed over email, face to face or in front of a gathering, can convey the affirmation that many look for in their professional lives.

A death in the workplace can feel like a death in the family. The thing that matters is that not many businesses can manage the cost of time to grieve.

In the aftermath of a workplace loss, your team will require support and a way ahead. The sad reality is you won't have much time to move from catastrophe to strategy (Workopolis, 2017). Furthermore, your employees will depend on you to deal with the situation effectively and with sensitivity.

"It says a great deal regarding an organization how they treat an employee who dies," said Alan Kearns, career coach and founder of CareerJoy. "It's extremely vital how an organization behaves, explicitly in this situation, that they show tact, sympathy, and compassion to the employee, their family, and other workers. People are going to pay attention and know about what it implies."

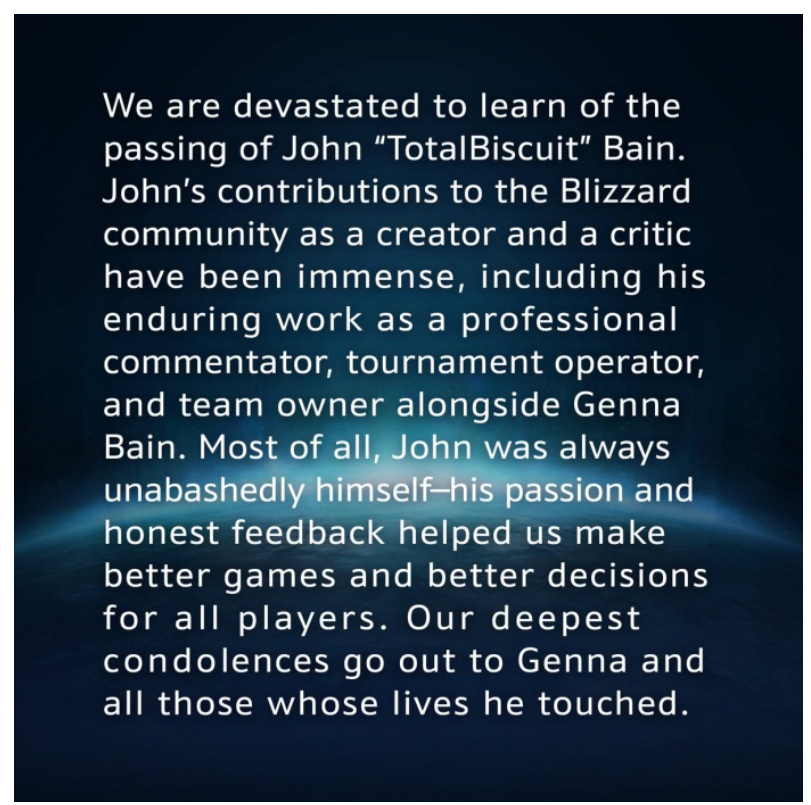

Figure 13. Appreciation and Expression of Sympathy Post

In this manner of posting an appreciation and sympathy post drags the audience into the company. It seems that the company is very compassionate to their employees and that is so attractive to the audience. This appreciation and sympathy post might not be a marketing strategy for the Blizzard as the post comes from their heart but truly, they gained fans out of it.

\section{Appreciation Post}

Having a customer appreciation program is an incredible method to help keep your customers happy and loyal, and stand out from your competitors (Martinuzzi, 2016).
Feeling unappreciated may provoke your customers to take their business elsewhere, for a company that will esteem their business. Customer appreciation is essential for some reasons. Above all else, it can help satisfy customers. A happy customer might be bound to return as well as spread the news about the company. This can help build your brand's reputation for being a good company to work with. In a time of globalization, impersonal online shopping and fast paced lifestyles, customer appreciation might be viewed as a lost art. Taking an old-school, back to basics approach to customer appreciation can help the company to stand out.

Everybody likes to feel valued. Customers are the same. It's important to recall, however, that with regards to customer appreciation, sincerity trumps tricks. Customer appreciation initiatives that are driven by a genuine desire to please your customers can be very successful. Real appreciation stems from a feeling that we really care for our customers. It's an indication of genuine gratitude for their business.

Nobody likes being in a one-way relationship - the kind where you're the only one committing any time or attention to keeping it going (McEachem, 2019). When you're always giving and never receiving, it's easy to feel unappreciated and undervalued.

The same is true in ecommerce. When customers are committed on the brand community, they want to feel like you care about them as people. Else, you risk them taking their business somewhere else.

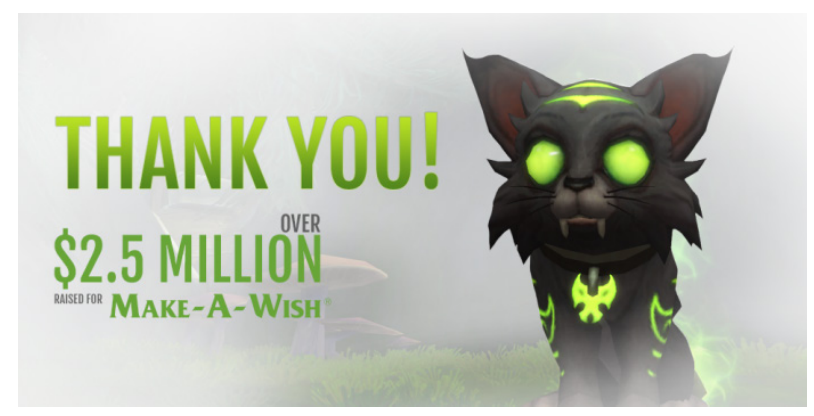

Figure 14. Appreciation Post

This is one of the greatest contributing factors to a customer choosing with another brand. While you may assume that most customers pick competitors based on the prices and products, $9 \%$ of customers leave because of what a competitor is offering while $60 \%$ of businesses have lost a customer since they feel a company is not interested in them. Clearly customers anticipate a little appreciation as an end-result of their business.

In order to avoid losing them, the simplest thing you can do to make a customer feel valued is to continually express gratitude toward them for being a part of the community. Through email communications, social messages, and handwritten notes, you can remind customers that you are aware of their business and appreciate it (McEacherm, 2019). 


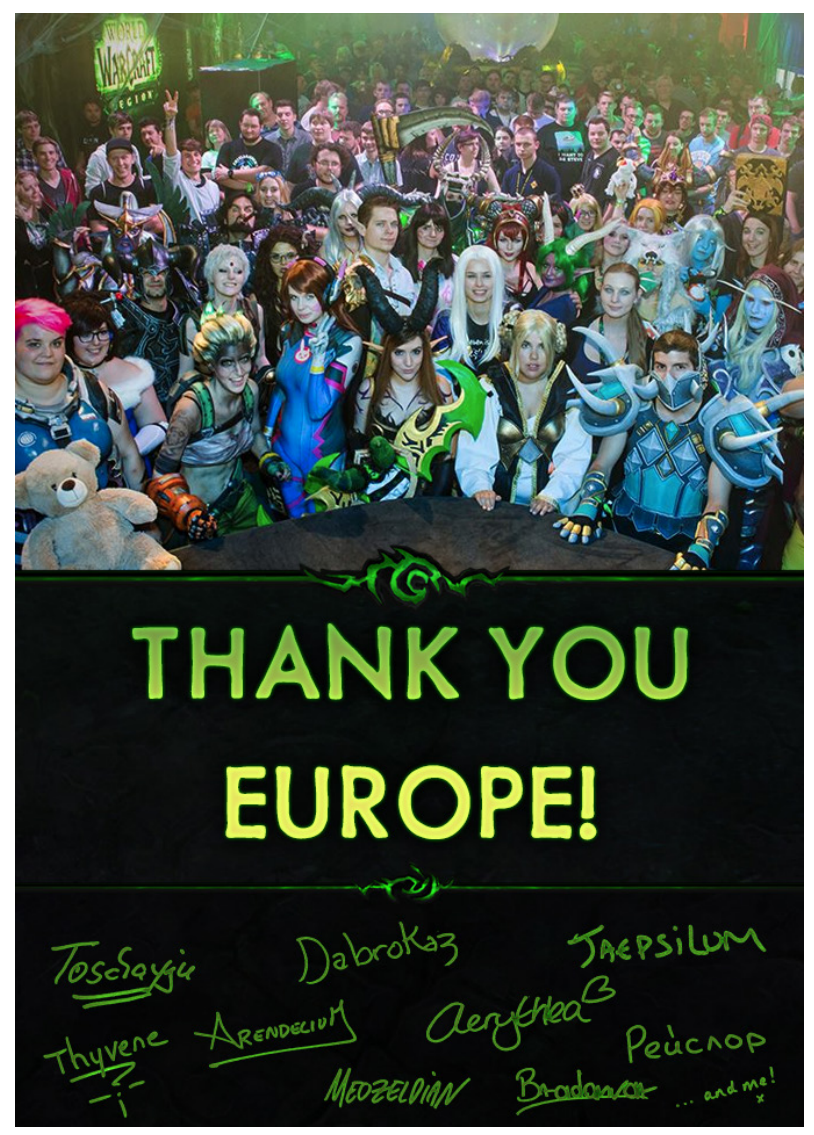

Figure 15. Appreciation Post

Through this love language (appreciation post) of Blizzard, customers are more interested to continue being with them. It just shows that there is a relationship between the company and its customers. In this generation that social media is very prone to all the people, posting an appreciation message is one of the ways in thanking customers for the support and also their appreciation to the business.

\subsection{How does the Language of Advertisements Influence People?}

B. Analysis of its Influence

1. Blogs

Blogs are everywhere on the web nowadays. There are millions of them, in all shapes and sizes, with no real rules.

A blog (another way to say "Web Log") is basically a journal or individual journal that is accessible on the web. Blogs are typically updated every day using software that permits individuals with little or no technical background to update and look after them.

The activity of updating a blog is called "blogging"; somebody who keeps a blog is a "blogger." A blog can be whatever the author needs it to be, as it gives people their own voice on the web. Consider it an everyday pulpit, a political soapbox, a collection of links, or somebody's private thoughts or memos to the world. In simple terms, a blog is a website updated on a continuous premise. New information appears at the top, so visitors can read what's new.

If you don't recognize Blizzard Entertainment by name, you definitely have known about what they have created: the enormously popular online games Warcraft, StarCraft, the Diablo series, Heroes of the Storm, Hearthstone and Overwatch. However, as prominent as their game are around the work, Blizzard Entertainment appears to be similarly as popular with their employees: the company has earned a general rating of 4.3 on Glassdoor and been named one of the Best Places to Work in 2018.

As you may expect, Blizzard Entertainment draws in a great deal of gamers - gamers who are enchanted that their perks package includes an employee discount on games and even a few freebies, as indicated by one previous representative. "Blizzard offers a great deal of discount/ freebies on their own product, which is pleasant on the grounds that it makes you feel like part of the family," he said.

With respect to that worker discount, it ranges "25 percent to 50 percent," as indicated by a current employee. "There are times where extensive discounts happen depending on the time of the year (Kramer, 2018).

And through blogging, they reached out more customers or gamers as they bring a lot of interested visitors to their blogs. These visitors are always keen to see new updates from Blizzard. Thus, blogs are one way to communicate to the gamers or customers about the updates and discounts that they may get. Also, these blogs enable the customers to voice out the things that they want to say. For example, a gamer showed his gratefulness by writing a letter. Therefore, the blogs of Blizzard are influential as they show the benefits of their products and it also allows customers to write their thoughts about them.

\section{Social Media}

Social media life has been defined as websites which permit profile creation and visibility of connections between users (Boyd and Ellison, 2008); web-based applications which give functionality to sharing, connections, gathering, conversation and profiles (Kietzmann et al., 2011). Social media has been referred to as "social media sites" (Diga and Kelleher, 2009), or a set of information technologies which encourage interactions and networking (Kapoor et al., 2017; Oestreicher-Singer and Zalmanson, 2013). In any case, there appears to be a wide understanding that Web2.0 technologies played a significant role in the development and adoption of social media (Wolf et al., n.d.).

\section{a. Twitter}

Twitter is a microblogging service of around six years of age, and has more than 41 million users as at July 2009 (Omofonmwan, 2012). And it has come up with the 
identification of new happenings. One of the events that made Twitter popular was the situation of USA airways jet that crashed into the Hudson River. The main photo of the accident actually appeared on twitter before any news media arrived. Essentially, the users in twitter follow others or are being followed. Not at all like other social networks sites, it doesn't necessarily to be reciprocated. In Twitter, as a follower, the user gets all the messages "known as tweet" from those the user follows. RT stands for retweet, '@' followed by a user identifier address the user, and '\#' followed by a word represents to a hashtag. The well-defined markup vocabulary combined with a short message, 140-character limit for each message. Thus, you read other people's tweets. The retweet method empowers users to spread and share information of their choice beyond the reach of the original tweet's followers.

The rise of streaming over the past long years has shifted video game marketing in a big way. According to one of the staffs of Blizzard, it is very important to have influencers involved not just with the marketing process of a game, but with the development as a whole. Influencers and pro-players of Blizzard titles are frequently invited to the company's campus in California, where they can test new updates and builds first, presumably before they go in the public test regions. And as Twitter has become an efficient platform for advertising companies as a new vast medium, it is obvious that finding influential Twitter users and measuring their influence are important. The Twitter pages of Blizzard provide a lot of news update and humorous posts that attract followers. Their creativity in making puns are shown in their Twitter pages. Thus, creates a good market.

\section{b. Facebook}

Facebook is a popular social networking site. Initially restricted to individuals with .edu email addresses, Facebook is presently accessible to anybody with a valid email address. A valid email address is one that is confirmed by Facebook - the user must get an email message at that address and click on a link in the message to demonstrate that it is a working address. Facebook members can join networks based on school affiliation, employers, and geographic regions. Facebook can be used for keeping track of friends both old and new. It is free to join and requires just that you be over than 13 years old and have a valid email address.

A Facebook page has numerous potential benefits for businesses. While some of these benefits are similar to having a website, a number are unique to Facebook.

Marketing activities that would cost a thousand of dollars through different channels can be used on Facebook for a small amount of the expense. This makes it ideal for small to medium businesses with a limited marketing budget. Bigger businesses can also trial marketing concepts and themes through Facebook before committing on greater campaigns.
Just as enabling you to post text, Facebook gives you a chance to upload pictures and videos from your business. This can be an incredible method to speak with customers and potential clients, enabling them to see the product or service without visiting your premises.

Customers can also post after-sales questions on the Facebook wall, and a staff can answer them there. This is frequently more effective than staff answering telephone calls, and enables different customers to read common questions and answers without moving toward you exclusively.

Blizzard also has Facebook pages in which they post updates about their games and so on. As one of the leading eSports games developers in the world, with the likes of World of Warcraft, Hearthstone and Overwatch to its name, Blizzard's choice to use Facebook Live as the platform to stream one of more obscure titles hints that there could be more to come from the social network in the gaming sector.

Having recently been broadcast, the content will presently stream live from the Heroes of the Storm Facebook page (which has barely short of two million followers). "We're also excited to work with Facebook this year to bring all the more live Heroes of the Dorm play to more viewers around the globe than ever before (McCarthy, 2017)." These are mainly the words of one of the employees of Blizzard. Therefore, Facebook becomes their platform for live streaming and they reach a lot of followers.

\subsection{Do These Advertisements have an Impact on Our Society and Cultural Values?}

Online video games have increased massive popularity in recent years. Video gaming has become a sport, a pastime and a hobby for many. Online gamers develop many social contacts through games. A large number of these contacts develop into lasting friendships. Video games have become one of the most well-known entertainment mediums alongside the movie and music industries during the last decade (Poyhtari, 2016).

A couple of years back video games turned into the most well-known media by overtaking movies in consumer spending on entertainment media (EGDF, 2011). Video game industry includes gaming hardware and games in both physical and electronic form for PC, consoles, cell phones and other gaming devices. The industry was estimated to be worth 67 billion dollars in 2012 and it has been anticipated to grow to 82 billion dollars by 2017 (Gaudiosi, 2012).

The social communities in games don't restrict themselves just to the internet. LAN parties are also very popular among gamers. In LAN-parties the gamers bring their own PC to the event venue where they take part in gaming and socializing with other gamers. In a many of these events there are also E-sports (electronic games) competitions where the gamers go up against one another. 
LAN-parties have integrated deeply in to the youth and popular culture. (Taylor and Witkowski, 2010.)

Blizzard Entertainment stands tall in the gaming industry, their sterling reputation almost unmarred by the failures and embarrassments that few companies have managed to avoid (Duffy, 2003). Their short history is characterized by both the commercial success and artistic accomplishment of every one of their games. No place is Blizzard's incredible success story more evident than in the field of Real Time Strategy (RTS) games, an area where they continue to dominate. Despite the fact that Blizzard did not pioneer the RTS format, they revolutionized and refined the concept into "a core genre of PC gaming" with their Warcraft and Starcraft titles (PC Retroview: Dune II).

Computer video games should be a leisure activity through which a person can escape from the real world. Bellman (2014) says that for advertisers the best time to utilize their manipulative tools is when an individual believes that the person is safe and can't be influenced by the outside world. This effect is bound to show up while playing games. Video games cannot be considered as a pure leisure activity any longer because they have become a "gold mine" for advertisers. Thus, advertisers and marketers use this leisure time to gain customers and money for their business (Stepanova, 2016).

However, even if there are negative impacts about gaming advertisements to the society, there are also good about it. In spite of the fact that numerous video games are made with the overarching reason for offering the player a source of entertainment and stimuli, video games having a place with a classification regularly called 'edutainment' (education and entertainment) are turning into a growing part of the industry. These games are regularly made with the aim of being placed in a classroom in order to help students better connect and comprehend traditional subject material, like to science, math, and history (Baker, 2014).

Cultural productions, for example, entertainment media, have been discussed about in numerous cultural studies throughout the years. Some of them have addressed the differences between eastern and western values and beliefs that are being reflected in cultural structures, symbols, and expressions. A collection of essays in "Multiple Modernities" (Lau, 2003) records observations and explores modern and postmodern popular culture as a generator, just as a result of various cultural forces (Yi Ngai, 2005). It demonstrates that films and other forms of well-known culture are culturally reflexive media, which are strongly shaped by local context and conditions. "

Fundamental differences, for example, cultural norms, values, and beliefs can impact the success or failure of the transnational-ability of cultural products. For video games, a compelling game experience is related to emotional responses and perceptions. How much "fun" players are having is very much depended on each person, and their perceptions of "fun". Such preferences and attitudes are based heavily on personality, just as societal values that are imbedded in a culture. (Aoyama and Izushi, 2003; Jesse, 2001; Carless, 2004)

In this manner, it implies that video games establish cultural differences similar to those traditional, non-interactive entertainment media. Moreover, in understanding cultural implications can be beneficial for non-entertainment or "serious" games, for example, education and training programs. Effective delivery of content is important; however, learning experience can become more engaging through uplifting enjoyment.

\section{Conclusions}

Advertisements are a popular thing which people watch or read each day, but they are designed in a cautious way to pull in clients (Sase \& Abdelaal, 2014). Advertisements can be analyzed according to different theoretical work in order so as to be able to understand them. The positioning of the actors of the advertisement gives a certain message, in some cases it is related to power or dominancy. The colors utilized additionally reflect another message; other than the language used which also reflects a brief and strong message. Furthermore, advertisements need further studies to go deeper in understanding how they are designed to attract customers.

Based on the data analysis of the advertisements, it can be concluded that advertisers of Blizzard's Entertainment use various linguistic devices such as catchy slogans or poems, puns, time-bound greetings, riddle discourse, ellipsis, etc. The researcher observed that the informal style of advertising language predominates over the formal style. Moreover, advertisements are the best specimen to witness the social changes and trends that influence the masses. The writer's criterion for making an advertisement is the best form of deep analysis and strong command over the customers and market. Language is a weapon that triggers off human mind. The advertiser knows the nerves of the society and its subjects, and accordingly plans the advertising messages and casts a spell over writing. Writers think about the demeanor of a buyer and utilize language with the assurance that it will bring out the ideal reaction to what they need to sell. The writers are continuously looking for more intelligent approaches to grind the customer to part with money.

Furthermore, with the coming of Internet technologies we just haven't seen a similar knock on impact where advertisements are becoming part of some popular culture discussions. Therefore, video game advertisements of Blizzard Entertainment have great impacts not just socially but culturally. Advertising itself is broadly considered to be a contribution to culture. Advertising is integrated into a lot of products. However, there is just a little room left outside the consumption economy, in which culture and art can develop independently and where alternative values can be expressed. 
In spite of the fact that it is hard to distinguish what makes an advertising strategy that will appeal to all consumers, the findings of this study suggest that the use of linguistic features is a successful strategy in print advertising. Besides, a few specific devices, acting either alone or in combination with one another, have proven to be particularly effective at increasing consumer appreciation for, enthusiasm for, and recall of an advertisement and could help advertisers to grow progressively effective advertisement campaigns.

Finally, the researcher can say that this paper implies that advertisements proved to be one of the most effective tools to influence consumer purchasing decision, at least in Blizzard Entertainment's marketing communications. However, since the paper doesn't have enough examples and materials, it is recommended that future researchers will focus on the outcomes of online advertisements on the rural areas wherein social media is not that popular nor taken advantage of. In this way, the researcher can truly see the impact and influence to the society. Thus, this paper is a support to the upcoming researches related to social media, advertising and marketing strategies.

\section{Acknowledgement}

It's really nice to wake up in the morning realizing that God has given me another day to finish this paper. Thus, I only thank my God for always giving me the strength to keep going.

\section{REFERENCES}

[1] Arroyo, B. (2014) The discourse analysis of cosmetic advertising: max factor's discursive development from 1940 to the present. Universidad de Valladolid.

[2] Baker, C. (2014) Video games: their effect on society and how we must modernize our pedagogy for students of the digital age. Virginia Commonwealth University.

[3] Bregenhoj, A. (2018) The riddle: form and performance. University of Turku.

[4] Bregenhoj, A. (2001) Riddles: perspectives on the use, function and challenge in a folklore genre. Helsinki. Finnish Literature Society.

[5] Cumming, R. (no date) Speech rhythm: the language specific integration of pitch and duration. Downing College.

[6] Dasgupta, S. (2015) Discourse analysis of telecom ads: a critical insight from the applied linguistics perspective. Brac University.

[7] Duffy, S. (2003) A perfect storm: StarCraft as a window into Blizzard's design philosophy and success.

[8] Fairclough, N. (1995) Critical discourse analysis. Available a thttps://www.hse.ru/data/2015/04/13/1094925608/Critical

\section{\%20discourse\%20analysis_THEORY_FAIRCLOUGH.pdf}

[9] Gee, J. (1999) An introduction to discourse analysis: theory and method. Library of Congress Cataloging in Publication Data.

[10] Goddard, A. (2001) The language of advertising. Library of Congress Cataloging in Publication Data.

[11] Gully, A. (no date) The discourse of Arabic advertising: preliminary investigations. Journal of Arabic and Islamic Studies.

[12] Jawad, B. (2016) Ellipsis in English. University of Al-Qadissiya.

[13] Korcak, J. (2012) World play in advertising: a linguistic analysis. Tomas Bata University in Zlin.

[14] Kramer, J. (2018) What's it really like to work at Blizzard Entertainment. Available at https://www.glassdoor.com/blo g/blizzard-entertainment/

[15] Lawson, A. (2008) One-on-one with Obama: an analysis. The University of Birmingham.

[16] Martinuzzi, B. (2016) The importance of customer appreciation - and 12 ways to do it right. Available at https://www.americanexpress.com/en-us/business/trends-an $\mathrm{d}$-insights/articles/the-importance-of-customer-appreciation /

[17] McCarthy, J. (2017) Facebook live snags Blizzard's heroes of the storm eSport broadcast from ESPN. Available at https://www.thedrum.com/news/2017/01/25/facebook-livesnags-blizzard-s-heroes-the-storm-esport-broadcast-espn

[18] McEachern, A. (2019) Why you should always be thanking customers. Available at https://blog.smile.io/thanking-custo mers

[19] McKee, A. (2003) Textual analysis: a beginner's guide. University of Technology Sydney.

[20] Nakilcioglu, I. (2013) The effects of font type choosing on visual perception and visual communication. Afyon Kocatepe University. Online Journal of Art and Design.

[21] Ngai, A. (2005) Cultural influences on video games: players' preferences in narrative and game-play. Ontario. University of Waterloo.

[22] Okan, Z. (2017) Critical discourse analysis of advertising: implications for language teacher education. International Journal of Languages' Education and Teaching.

[23] Omofonmwan, N. (2012) What is Twitter, a social network or a news media? Hochschule Furtwangen University.

[24] Poyhtari, A. (2016) Social gaming in online games. University of Oulu.

[25] Sase, A. \& Abdelaal, N. (2014) Advertisement analysis: a comparative critical study. Australia. Australian International Academic Center.

[26] Stepanova, V. (2016) Commercial influence on the video game industry: symbiotic relationships and their effects. University of New York in Prague.

[27] Vasiloia, M. (no date) Linguistic features of the language of 
advertising. George Bacovia University.

[28] Available at https://news.blizzard.com/en-gb/diablo3 (Acce ssed: 20 April 2019).

[29] Available at https://news.blizzard.com/en-gb/hearthstone (Accessed: 20 April 2019).

[30] Available at https://twitter.com/Warcraft (Accessed: 20 April 2019).

[31] Available athttps://news.blizzard.com/en-gb/world-of-warc raft (Accessed: 20 April 2019).

[32] Available at https://twitter.com/Diablo (Accessed: 20 April 2019).

[33] Available at https://twitter.com/PlayHearthstone (Accessed: 20 April 2019).
[34] Available at https://twitter.com/Blizzard_Ent (Accessed: 20 April 2019).

[35] Available at https://web.facebook.com/WorldofWarcraft.eu /?brand redir $=138011799033 \&$ rdc $=1 \&$ rdr (Accessed: 20 April 2019).

[36] Available at https://web.facebook.com/Hearthstone.en/?bra nd_redir=498467596862680\&_rdc=1\&_rdr (Accessed: 20 April 2019).

[37] Available at https://web.facebook.com/Diablo.eu/?brand re dir $=157102206640 \& \_r d c=1 \& \_r d r$ (Accessed: 20 April 2019).

[38] Available at https://web.facebook.com/Blizzard/?_rdc=1\&_ rdr (Accessed: 20 April 2019). 